\title{
The Research on The Time-Dependent Evaluation Model of Space Debris Removal
}

\author{
Junjie Zhou ${ }^{1, a}$ \\ ${ }^{1}$ North China Electric Power University, Baoding, China \\ a2174745153@qq.com
}

Keywords: Time-Dependent Alternatives Evaluation Model, space debris, Borda Count Method

\begin{abstract}
In this paper, we build the Time-Dependent Alternatives Evaluation Model to determine the best alternative or combination of alternatives to remove the space debris. The model is consist of three small models, such as Costs, Risks and Benefits models which are time-dependent models. After consulting some researches, we discover five basic alternatives: S1, S2, S3, S4, S5. Using measure parameters of the alternatives, we obtain specific quantities of Costs, Risks and Benefits. Considering the factors, we qualitatively analyze five alternatives by Borda Count Method. S2 (ground-based laser) might be the best alternative.
\end{abstract}

\section{Introduction}

There are a number of alternatives to remove the space debris including small, space-based water jets and high energy lasers etc. To get the best alternative, it is necessary to put forward several alternatives by evaluating some important factors such as costs, risks and benefits. Then, the best alternative or the combination of alternatives can be determined. In addition to evaluate directly by the factors, the method of approaching ideal point is performed.

What's more, time is an significant variable. In this paper, time express different year, it is discrete. The important factors such as costs, risks and benefits are influenced by time directly or indirectly. Therefore, we establish a time-dependent alternatives evaluation model.

\section{Time-Dependent Alternatives Evaluation Model}

\section{Alternatives of space debris removal:}

By referring to information, some technical alternatives are collected: space-based laser(S1), ground-based laser(S2), solar sail(S3), resistance-increased device(S4), electric cable(S5). The specific alternative or combination is shown as follows:

Table 1: the character of different alternatives

\begin{tabular}{c|c|c|c|c|c|c}
\hline Alternatives & Target orbit & $\begin{array}{c}\text { Removing } \\
\text { ability }\end{array}$ & Volume & Quality & $\begin{array}{c}\text { Spending } \\
\text { of time }\end{array}$ & Lifetime \\
\hline S1 & LEO/MEO/GEO & 0.01 & 50 & 200 & 15 & 20 \\
S2 & LEO & 0.01 & 0 & 0 & 15 & 50 \\
S3 & LEO/MEO/GEO & 3 & 80 & 250 & 80 & 10 \\
S4 & LEO & 2 & 80 & 300 & 120 & 10 \\
S5 & LEO & 2 & 200 & 300 & 120 & 5 \\
S2+S3 & LEO/MEO/GEO & 10 & 80 & 250 & 15 & 40 \\
S3+S5 & LEO/MEO/GEO & 10 & 280 & 550 & 100 & 15 \\
S1+S5 & LEO/MEO/GEO & 5 & 250 & 500 & 30 & 25 \\
S1+S2 & LEO/MEO/GEO & 1 & 50 & 200 & 7 & 40 \\
\hline
\end{tabular}

We define $C(t), R(t), \quad B(t)$ stand for the time functions about costs, risks, benefits respectively. And then, we should establish three models to analysis and evaluate these alternatives. 


\section{Risks Model:}

According to the model, risks should meet the equation as follows:

$$
R(t)=e^{(j+P(t))}+G(q) g M ;
$$

Where $p(t)$ is the probability of a satellite being hit by space debris within a year. $j$ is a collision parameter. So $e^{(j+P(t))}$ is used to measure the risk of debris impact to the alternatives. $G(q)$ is a function of the satellite's fault.

There are three orbits that we should consider. If we regard their heights are $5000 \mathrm{~km}, 6000 \mathrm{~km}$ and $36000 \mathrm{~km}$, then discuss the probability of collision happened in different orbits. From model I, diameters of most of debris are less than $10 \mathrm{~cm}$. In the paper we only consider most debris.

\section{Costs Model:}

According to the model, costs should meet the equation as follows:

$$
C(t)=m(i) g A g h+\text { round }\left[\frac{v_{1}}{v_{2}}\right] g E(i)+D g V \int_{0}^{1} F d l g_{d} \text { ground }\left[\frac{v_{1}}{v_{2}}\right]+k\left[t_{d} \text { ground }\left[\frac{v_{1}}{v_{2}}\right] / Y\right](2)
$$

Among them, $m(i)$ represents the quality of the satellite in different alternatives. $A$ is the transmission coefficient, $h$ is the height in different alternatives. So the physical meaning of $m(i) g A g h$ refers to the costs that a company launch a satellite to remove the debris.

$v_{1}$ is the total area where debris need to be removed, and $v_{2}$ is capability of removing the debris(the area where the satellite can remove all debris once). If $E(i)$ is the cost that debris to be removed once, round $\left[\frac{v_{1}}{v_{2}}\right]$ indicates the number of times to remove. Then round $\left[\frac{v_{1}}{v_{2}}\right] \mathrm{gE}(i)$ is the cost using alternative $i$.

To get $E(i)$, we scan the paper from others[1]:

$$
\begin{aligned}
& E(i)=\frac{V_{\text {cost }}}{N_{\text {total }}} ; \\
& N_{\text {total }}=N_{\text {cata } \log e} \mathrm{~g} \frac{F}{F^{\prime}} ;
\end{aligned}
$$

Where $N_{\text {total }}=402000, F=0.0084,0.0032,0.0053$ and $\quad F^{\prime}=0.0032,0.0027,0.0016 . F \quad$ and $F^{\prime}$ are obtained from model I. $V_{\text {cost }}$ is the fixed cost of private firm. By calculation, $V_{\text {cost }}$ equals to $\$ 164.349$, $\$ 231.923$ and $\$ 315.779$ in different orbits.

$k\left[t_{d}\right.$ ground $\left.\left[\frac{v_{1}}{v_{2}}\right] / Y\right]$ expresses the depreciated cost, $k$ is a depreciated parameter. And $\mathrm{Y}$ is lifetime of a satellite.

\section{Benefits Model:}

As the model shows, benefits should meet the equation as follows:

$$
B(t)=\sum_{i=1}^{2} E_{i} \text {; }
$$

In this model, benefits are divided into two parts, $E(1)$ and $E(2)$ : 


$$
E(i)=\left\{\begin{array}{c}
E(1)=E_{m}(h) g^{\alpha F} \\
E(2)=\text { Cground }\left[\frac{v 1}{v 2}\right] \mathrm{g} E(i)
\end{array} ;\right.
$$

Where $E(1)$ represents the space value of different heights. $E(2)$ is the private firm's charges of removing space debris. In $E(1), E_{m}(h)$ is a function of space value. $C$ is called value coefficient. $e^{\alpha F}$ represents the influence to the space value caused by the flux of space debris. $E(i)$ is the unit price of space removing. round $\left[\frac{v 1}{v 2}\right]$ indicates the number of times to remove the debris.

\section{Borda Count Model:}

Solutions based on risks, costs and benefits are obtained, we can use Borda Count model [2] to make the qualitative order of the multiple attribute solutions. Then We can determine the optimal ordering.

The basic idea of the model is to establish an evaluation set $D=\left\{d_{1}, d_{2}, \mathrm{~L} d_{n}\right\}$, where $d_{n}$ is the corresponding ranking. Every $d_{n}$ weighs differently. $w\left(d_{i}\right)$ is used to express it. Borda count is the sum of all $w\left(d_{i}\right)$ :

$$
B(i)=\sum_{i=1}^{n} w\left(d_{i}\right) D(i), i=1,2, \mathrm{~L} n \text {; }
$$

By contrast of Borda count, we get the best order.

\section{Conclusions:}

According to the result of Borda count model, we obtain table 2and table 3.

Table 2: The rank of each character

\begin{tabular}{c|c|c|c|c}
\hline Alternatives & Risks & Costs & Benefits & $\begin{array}{c}\text { Borda } \\
\text { Count }\end{array}$ \\
\hline S1 & 2 & 3 & 1 & 18.33 \\
S2 & 3 & 1 & 2 & 16.33 \\
S3 & 9 & 5 & 6 & 4.33 \\
S4 & 4 & 6 & 7 & 6 \\
S5 & 5 & 7 & 3 & 7.66 \\
\hline \multicolumn{3}{c}{ Table 3: The Borda counter } \\
\cline { 2 - 4 } & \multicolumn{3}{|c|}{ Alternatives } & Borda Count \\
\cline { 2 - 3 } & S1 & 18.33 \\
& S2 & 16.33 \\
& S5 & 7.66 \\
S3 & 4.33 \\
\cline { 2 - 4 } & S3
\end{tabular}

Ranking these Borda values, we get the best order in different orbits(from the best to the worst ):

$$
S 1 \rightarrow S 2 \rightarrow S 5 \rightarrow S 4 \rightarrow S 3
$$

Finally, we can find the best alternative is S1. It means space-based laser is the best alternative to removing space debris if we take risk, cost and benefit into consideration.

\section{Reference:}

[1]Leonard Vance, Allan Mense, "Value-analysis for orbital-debris-removal”, Jauary,32013 
[2] Dong xiaobo, "The application of Borda method in choosing the best Personage", 1996.01:19-22

[3] Huang JianGuo, Han JianWei, Li HongWei, "Investigation on the surface damage to solar cells by impacts of space micro-debris on low earth orbit"2008

[4] Li Yiyong, Li Zhi, Chen Yong, "Analysis and Applications on Sunlighting Characteristics of Space Objects",2014 